\title{
Understanding double-resonant Raman scattering in chiral carbon nanotubes: Diameter and energy dependence of the $D$ mode
}

\author{
Felix Herziger,* Asmus Vierck, Jan Laudenbach, and Janina Maultzsch \\ Institut für Festkörperphysik, Technische Universität Berlin, Hardenbergstr. 36, 10623 Berlin, Germany
}

(Dated: September 20, 2018)

\begin{abstract}
We present a theoretical model to describe the double-resonant scattering process in arbitrary carbon nanotubes. We use this approach to investigate the defect-induced $D$ mode in CNTs and unravel the dependence of the $D$-mode frequency on the CNT diameter and on the energy of the resonant optical transition. Our approach is based on the symmetry of the hexagonal lattice and geometric considerations, hence the method is independent of the exact model that is chosen to describe the electronic band structure or the phonon dispersion. We finally clarify the diameter dependence of this Raman mode that was controversely discussed in the past and demonstrate that, depending on the experimental conditions, in general two different dependencies can be measured. We also prove that carbon nanotubes with arbitrary chiral index can exhibit a $D$ mode in their Raman spectrum, in contrast to previous symmetry-based arguments. Furthermore, we give a direct quantification of the curvature-induced phonon frequency corrections of the $D$-mode in carbon nanotubes with respect to graphite.
\end{abstract}

PACS numbers: 61.48.De,78.67.Ch,63.22.Gh,78.30.Na

\section{INTRODUCTION}

Carbon materials such as graphene and single-walled carbon nanotubes (CNTs) attracted much scientific interest in the past decades ${ }^{1,2}$. Their extraordinary properties made them a perfect candidate for applications in novel electronic devices such as transistors or sensors ${ }^{3-5}$. Here, functionalized systems are most important, since their properties can be designed and manipulated as preferred $^{6-8}$. The success of a functionalization is often monitored by investigating the increase of the Raman $D$-mode intensity ${ }^{9}$. This mode stems from TO phonons around the $K$ points of the graphene Brillouin zone and is activated by breaking the translational invariance, thus relieving selection rules ${ }^{10}$. Although the defect-related origin of the $D$ mode was known for a long time, the underlying Raman processes remained unclear $^{11}$. Thomsen and Reich solved this longstanding question by introducing the double-resonant scattering theory for graphitic systems ${ }^{12}$, explaining the anomalous dispersion of certain Raman modes, e.g., the $D$ and $2 D$ modes, with laser excitation energy ${ }^{13}$. However, the double-resonant $D$ mode was thought to exist only in carbon nanotubes that satisfy $k_{F}=K,{ }^{14}$ where $k_{F}$ is the reciprocal vector of the singularity in the joint density of states. This relation is particularly true for armchair carbon nanotubes and other metallic tubes with $\mathcal{R}=3$, i.e., $\left(n_{1}-n_{2}\right) /(3 n)=$ integer $^{1,15}$. In contrast, semiconducting carbon nanotubes were predicted to not exhibit a $D$ mode with the same systematic excitation energy dependence of its frequency ${ }^{14}$. However, recent experiments on semiconducting CNT samples enriched with single chiral indices demonstrated the existence of a $D$ mode in CNTs that do not satisfy the aforementioned restriction ${ }^{16}$. Furthermore, there is an ongoing discussion about the dependence of the $D$-mode frequency in CNTs on the tube diameter and the transition energy. During the past decade many different explanations were postulated and controversely discussed. For instance, Souza Filho et al. ${ }^{17}$ proposed an inverse diameter dependence of the $D$-mode frequencies, whereas Refs. 18 and 19 claimed a dependence proportional to the tube diameter. Thus, the systematics of these scattering process is still not fully understood and needs clarification.

In this work, we derive a universal, geometrical model that describes the dependence of the resonant phonon wave vector in the double-resonant scattering process on the tube diameter and the transition energy of the process for all tubes with arbitrary chiral indices, rejecting the previously predicted restriction of the $D$ mode to particular CNTs. Our model is based on the symmetry of the hexagonal lattice only and is thus universally valid, no matter which approximation for the electronic band structure or phonon dispersion is used. We apply this model to investigate the diameter dependence of the $D$ mode in CNTs for the resonant optical transition, which is still under controverse discussion. We will point out that, depending on the specific experimental conditions, in general two different diameter dependencies can be observed. Furthermore, we will highlight the importance of nanotube curvature effects on the phonon frequencies to explain the experimentally observed dispersion of the $D$ mode. Finally, we derive a diameter correction for the $D$-mode frequencies in carbon nanotubes with respect to the $D$-mode frequency in graphite.

\section{SIMULATION DETAILS}

All geometric considerations in this paper are based on the symmetry operations of the hexagonal lattice of graphene. Hence, within the framework of zone-folding, the results do not depend on the choice of the model to describe the electronic band structure or the phonon 


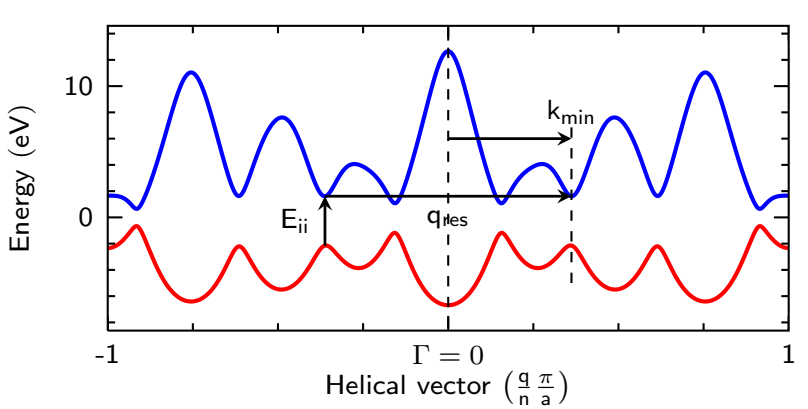

FIG. 1. Illustration of the double-resonant scattering process between two energetically equivalent minima in the helical band structure of a $(5,3)$-tube. The transition energy is labeled as $E_{\mathrm{ii}}$, the position of the electronic minimum as $k_{\min }$, and the resonant phonon wave vector as $q_{\mathrm{res}}$. The translational period is denoted with $a ; q$ reflects the subbands in linear quantum numbers.

dispersion.

The calculcations of electronic band structures and phonon dispersions in this work are based on the POLSym code in the sixth-nearest-neighbor approximation ${ }^{20}$. This package uses the modified group projector technique and includes curvature effects in the calculations of the band energies and phonon frequencies. Electronic bands and phonon dispersions are calculated for all 274 chiral tubes with diameters between 5 and $25 \AA$. Furthermore, we used an experimental graphite phonon dispersion from Ref. 21 for an alternative calculation of the $D$-mode frequencies and for comparison with the POLSym-derived frequencies.

We model the double-resonant scattering process in carbon nanotubes by assuming that scattering occurs mostly between equivalent minima in the electronic bandstructure (compare Fig. 1) ${ }^{16}$. As demonstrated by previous works, the double-resonance process is dominated by the incoming resonance with excitonic transitions at vanHove singularities in the electronic bandstructure ${ }^{22,23}$, justifying our assumption. Furthermore, we assume that the phonon frequency $\hbar \omega_{\text {phonon }}=0 \mathrm{meV}$, as the resonant phonon wave-vectors in the double-resonance process depend only weakly on the energy of the involved phonons $^{24}$. These assumptions do not affect the general validity of our results in this work.

\section{RESULTS AND DISCUSSION}

As we mainly consider chiral carbon nanotubes, it is convenient to use the helical representation of the wave vector and electronic bands for the following analysis ${ }^{25,26}$. In the zone-folding approach, the electronic band structure in helical quantum numbers is given by the cut of the helical wave vector $\widetilde{\mathbf{k}}_{z}$ with the electronic $\pi$ and $\pi^{*}$ bands of graphene along its path in reciprocal

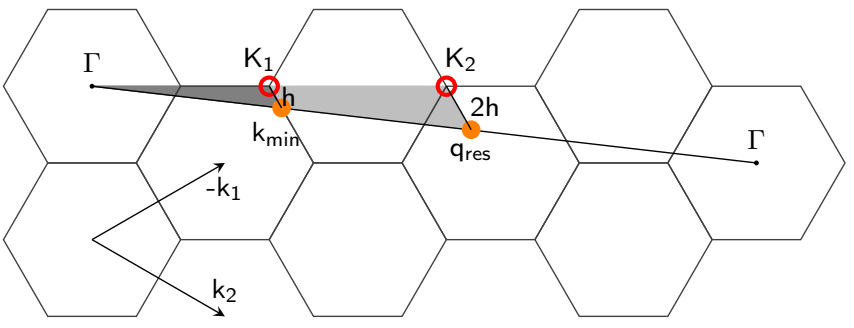

FIG. 2. Illustration of the dependence between the electronic transition and the resonant phonon wave-vector for a coprime tube, i.e., $n=1$. The black solid line denotes the helical vector of a $(3,2)$-tube, the full orange circles mark a minimum in the electronic band structure $\left(k_{\min }\right)$ and the corresponding resonant phonon wave-vector length $\left(q_{\text {res }}\right)$, which is twice $k_{\text {min }}$. The open red circles denote the closest $K$ points to $k_{\text {min }}$ and $q_{\text {res }}$, respectively. As can be seen easily, the distance $h=K_{1}-k_{\min }$ is always twice the distance $K_{2}-q_{\text {res }}$.

space. The helical wave vector is given by

$$
\widetilde{\mathbf{k}}_{z}=\frac{1}{n}\left(-n_{2} \mathbf{k}_{1}+n_{1} \mathbf{k}_{2}\right)+\Delta \mathbf{k}_{1}\left(n_{1}, n_{2}\right)+\Delta \mathbf{k}_{2}\left(n_{1}, n_{2}\right),
$$

where $n_{1}$ and $n_{2}$ are the chiral indices of the CNT and $n$ is the greatest common divisor of $\left(n_{1}, n_{2}\right)$. $\mathbf{k}_{1}$ and $\mathbf{k}_{2}$ are the unit vectors in graphene's reciprocal space (compare Fig. 2). The $\Delta \mathbf{k}_{i}$ describe the distance in reciprocal space of the $i$ th subband from the subband containing the $\Gamma$ point of graphene and is non-zero only if $n>1$. For a more detailed derivation of the helical wave vector $\widetilde{\mathbf{k}}_{z}$ and an introduction to the concept of helical zone-folding we refer the reader to the Supplementary Material ${ }^{27}$.

The $D$ mode results from a double-resonant intervalley scattering process, including a transverse optical (TO) phonon and a defect ${ }^{12,14}$. In Figure 2 we show the systematics of the $D$-mode scattering process for a $(3,2)$ tube. Our approach is of course valid for all other tubes having more than one subband (see Supplementary Material). Without loss of generality, we assume that the minimum in the band structure of a CNT occurs at the position where the helical vector crosses a $K-M-K^{\prime}$ high-symmetry line. The minimum shall have the $k$ vector $k_{\min }$ (measured from the $\Gamma$ point), hence the resonant phonon wave vector in the $D$-mode scattering process has the length $q_{\text {res }}=2 k_{\text {min }}$. This implies the socalled $q_{\text {res }} \approx 2 k_{\text {min }}$ rule. We label the $K$ point closest to the electronic minimum as $K_{1}$ and refer to its distance to $k_{\min }$ as $h$. In general, the distance $h$ is a measure for the transition energy, i.e., a smaller value for $h$ means a lower energy and vice versa. The three points $\Gamma, k_{\min }$, and $K_{1}$ form a triangle, which is indicated by the dark-gray area in Fig. 2. By similarity transformation of this triangle, we obtain the light-gray triangle formed by the points $\Gamma$, $q_{\text {res }}$, and $K_{2}$. It is an intrinsic property of the hexagonal lattice that twice the distance between $\Gamma$ and a $K$ point, is again a $K$ point $\left(K_{2}\right)$. If $K_{1}$ is the closest $K$ point to $k_{\min }$, then $K_{2}$ is the closest $K$ point to $q_{\text {res }}$. Since all sides of the larger triangle were doubled, the distance 

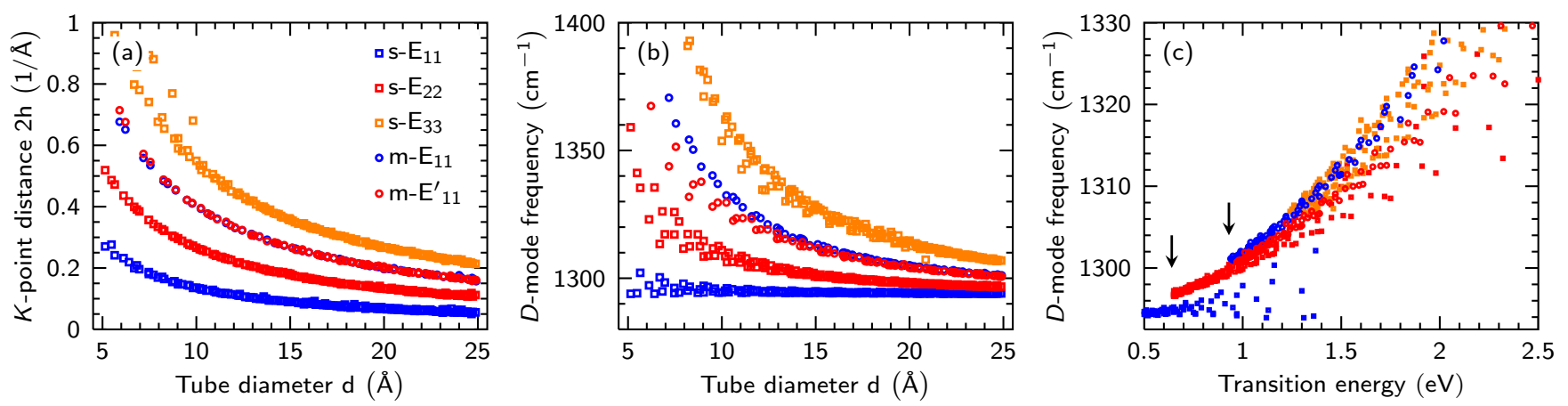

FIG. 3. (a) Distance between the resonant phonon wave-vector and the closest $K$ point as a function of the tube diameter for the resonantly enhanced scattering process obtained by a sixth-nearest neighbor tight-binding model for electronic band structures and phonon dispersions. (b) Calculated $D$-mode frequency $\omega_{\mathrm{D}}$ as a function of the tube diameter. (c) Calculated $D$-mode frequency as a function of the laser energy. The experimentally observed jumps between the different transitions are indicated.

$K_{2}-q_{\text {res }}$ is now twice the distance $h$. This means that an electronic transition with a distance $h$ from $K_{1}$ results in a resonant phonon vector with a distance $2 h$ from $K_{2}$ (at the same angle with respect to the closest $K$ point). Thus, $h$ and $2 h$ are indicative for the energy of the transition and the $D$-mode frequency, i.e., a larger value $2 h$ in general means a larger TO phonon frequency and vice versa. From this we can deduce two important results: First, tubes with large diameters and thus lower transition energies exhibit a systematically lower $D$-mode frequency for the resonantly enhanced transition compared to small-diameter tubes. Second, different transitions in a CNT exhibit different $D$-mode frequencies. Although these results seem trivial at a first glance, they enable a deeper understanding of the $D$ mode in CNTs.

If we now also consider trigonal warping effects in the electronic bandstructure, the electronic minimum may not be exactly on the $K-M-K^{\prime}$ high-symmetry direction, but slightly shifted away. This would lead to a slightly different distance $h$ and angle between $k_{\min }$ and $K_{1}$. Nevertheless, the distance between the resonant phonon wave vector $q_{\mathrm{res}}$ and $K_{2}$ is again $2 h$. Hence, this relation is independent from the exact position of the electronic minimum, which may differ depending on the model used in bandstructure calculations. Thus, our results are universally valid as long as zone-folding is an appropriate model to describe the properties of $\mathrm{CNTs}^{28-30}$.

Figure 3 (a) presents the calculated distance between the resonant phonon wave vector and the closest $K$ point for different optical transitions as a function of the tube diameter $d$. As can be seen, the distance $2 h$ decreases with a $1 / d$-dependence. Ergo, the resonantly enhanced TO phonon frequency decreases likewise. Furthermore, energetically higher transitions have a larger distance $2 h$ for the same tube diameter, corresponding to higher $D$ mode frequencies. Since the distance $2 h$ directly depends on the energy of the optical transition of a tube, we observe a close correspondence between the dependence shown in Fig. 3 (a) and the so-called Kataura plot ${ }^{31}$. By translating the distance $2 h$ into a phonon frequency using the POLSym-calculated phonon dispersions, we obtain the $D$-mode frequencies shown in Fig. 3 (b). As discussed before, a larger value for $2 h$ reflects a larger TO phonon frequency and vice versa, thus, the systematics of this plot again resemble the Kataura plot. We observe a decreasing $D$-mode frequency with increasing tube diameter. Furthermore, electronic transitions with a higher energy show an increased frequency.

If we plot the $D$-mode frequency as a function of the transition energy in Fig. 3(c), we observe an interesting feature in the $D$-mode dispersion. The dispersion of the branches is not continuous. In fact, a small jump in frequency between different electronic transitions is observed. This frequency jump has been reported previously in resonance Raman studies on enriched carbon nanotube samples ${ }^{23,32,33}$. As we discuss below, the observed jump is a direct consequence of curvature effects on the phonon frequencies and thus cannot be explained using a simple zone-folding approach. Considering a zone-folding third-nearest-neighbor tight-binding model without any curvature effects, only the helical vector would depend on $n_{1}$ and $n_{2}$, but not the electronic bands nor the phonon dispersion. Hence, two different tubes with different diameters but the same transition energy, i.e., same distance $h$, would exhibit the same $D$-mode frequency, i.e., same distance $2 h$. Thus, by neglecting curvature effects, the experimentally observed frequency jump cannot be explained. However, it is well-known that phonons in carbon nanotubes show a strong dependence on the tube diameter due to curvature effects and rehybridization of $\sigma$ and $\pi$ orbitals. For instance, in semiconducting tubes, the $\Gamma$-point TO phonon frequency $\left(G^{-}\right.$ mode) decreases with decreasing tube diameter ${ }^{34}$; a similar diameter dependence of the TO branch is expected around the $K$ point. Thus, two different tubes with different diameters but similar transition energies will not have the same $D$-mode frequency, i.e., the smaller tube has a lower frequency compared to the larger tube. Ergo, 

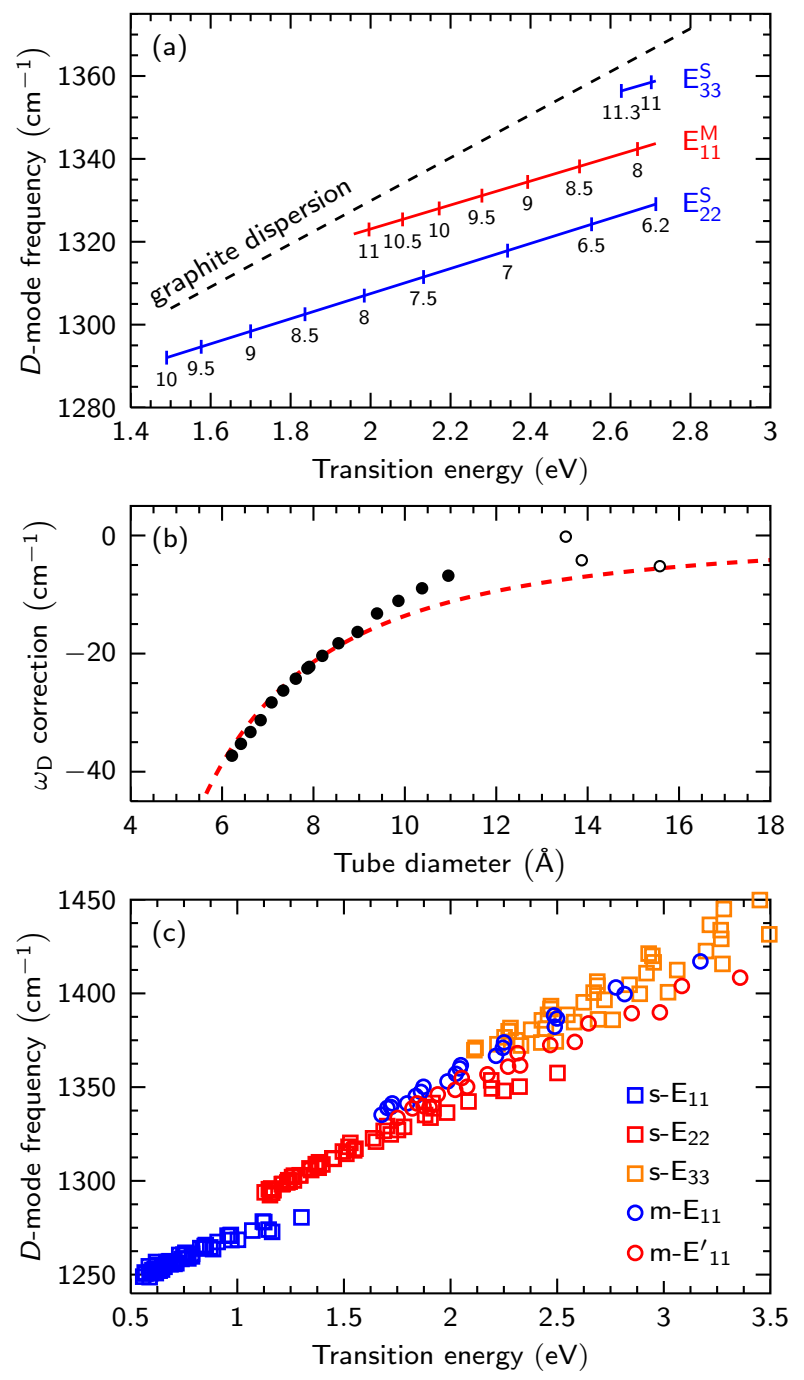

FIG. 4. (a) Schematized experimental data of the $D$ mode in carbon nanotubes (after Refs. 32 and 33). The solid lines denote fits to the experimental data for each transition; the numbers at each line correspond to the diameter of the tubes (in units of $\AA$ ). The dashed line indicates the $D$-mode dispersion in graphite. (b) $D$-mode frequency correction as a funtion of the tube diameter. The red, dashed line reflects a fit to the data points. Solid symbols were obtained from (a), open symbols are single-tube measurements from Refs. 32 and 33. (c) Curvature-corrected $D$-mode dispersion from applying Eq. (1) on the graphite dispersion. The experimentally observed discontinuity could be reproduced.

in a resonant measurement, one would observe a jump between different transition branches. In our calculations, this discontinuity is smaller than experimentally observed. Furthermore, $D$ mode from the $E_{11}^{S}$ transition shows nearly no dispersion with transition energy. We attribute these discrepancies to the fact that the Kohn anomaly and the region around the $K$ point in the TO phonon dispersion is not well approximated by the model for the phonon dispersion. Since the resonant phonons from the $E_{11}^{S}$ transition stem from a region close to the $K$ point, small deviations in the phonon dispersion directly influence the results and, in our case, lead to an overestimated $D$-mode frequency with nearly no dispersion. We expect a larger jump and a better correspondence to the experiments for calculations using a non-orthogonal tight-binding model or an ab-initio approach. Nevertheless, the existence of a jump can be reproduced in our simulations, as seen between $E_{11}^{S}$ and $E_{22}^{S}$ in Fig. 4 (c).

Next, we want to investigate the diameter distribution along a given optical transition $\mathrm{E}_{i i}$. The energy of a transition is inversely proportional to the CNT diameter, hence, when following a $D$-mode branch while decreasing the transition energy [see Fig. 3(c)], the diameter decreases.

The above presented results finally harmonize the different conclusions from various previous works where a decreasing $D$-mode frequency for a decreasing tube diameter was claimed ${ }^{18,19}$ or vice versa ${ }^{17}$. It is very important to point out that, depending on the experimental conditions, one can generally observe two different dispersion behaviors. By measuring the same optical transition for all tubes, e.g., the $\mathrm{E}_{22}$ transition, an $\propto 1 / d$-dependence will be observed due to the mentioned diameter distribution along the $\mathrm{E}_{22}$ branch. This would correspond to an experimental setup where the laser energy is tuned to measure every tube always at its resonance energy. By using only a single or just very few laser energies, a different behaviour is observed ${ }^{18,19}$. This experimental condition corresponds to a vertical cut through the dispersion relation shown in Fig. 3 (c). Along such a cut, a higher $D$-mode frequency corresponds to a higher tube diameter and a dependence proportional to $d$ is observed. Both cases are distinctly different and must be separated carefully.

To finalize our results, we want to give an explicit expression for the correction of the $D$-mode frequency as a function of the tube diameter, as a correction to the $D$ mode in graphite. As pointed out before, the phonon frequencies in CNTs show a strong dependence on the tube diameter. This dependence was shown both experimentally and theoretically for the $\mathrm{RBM}^{35-37}$, as well as for the $\mathrm{G}^{-}$mode $^{34}$. However, a direct evaluation of this dependence for the $D$ mode is still lacking. Starting from the schematized experimental $D$-mode dispersion shown in Fig. 4 (a) taken from Refs. 32 and 33 and the experimentally known dispersion of the $D$ mode in graphene and graphite ${ }^{10,38}$, we calculate the frequency difference between both dispersions and plot them as a function of the tube diameter. Since the $D$ mode in graphene and graphite does not include any curvature effects, the difference between those dispersions is a direct measurement of the diameter-dependent correction of the $D$-mode frequency. The result of this calculation is shown by filled and open circles in Fig. 4 (b). As expected, the frequency correction is always negative and converges to zero for increasing tube diameter. The dashed line reflects a fit to the data derived from a classical mechanical model (see 
Supplementary Material), using the formula:

$$
\Delta \omega_{D}(d)=A\left[\sqrt{1-\left(\frac{2.13 \AA}{d}\right)^{2}}-1\right] .
$$

Here, $d$ is the tube diameter and $A$ is a fit parameter obtained from fitting the data points in Fig. 4 (b) and was determined as $A=593 \mathrm{~cm}^{-1}$.

In the following, we will apply the above result to calculated $D$-mode frequencies obtained from a phonon dispersion that does not include curvature-induced effects. We use the calculated electronic minima and resonant phonon wave vectors from our sixth-nearest-neighbor model and obtain the resonant phonon frequencies from an experimentally measured TO phonon dispersion of graphite from Ref. 21. The CNT diameters in this calculation were chosen to fit the experimentally measured nanotubes from Refs. 32 and 33, i.e., only tubes with diameters between $6 \AA$ and $14 \AA$ were considered. Without curvature corrections, a linear dependence between transition energy and $D$-mode frequency is observed. By applying the diameter-dependent frequency correction according to Eq. (1), CNTs with smaller diameter observe a larger frequency downshift than nanotubes with large diameters with respect to the graphite $D$-mode dispersion. Thus, the slope of the $D$ mode dispersion in CNTs is reduced compared to the dispersion in graphite [compare Fig. 4(a)]. As a consequence, a discontinuity opens between different transitions. In our curvature-corrected $D$-mode dispersion from Fig. 4 (c), this discontinuity can be seen again most clearest between $E_{11}^{S}$ and $E_{22}^{S}$ (approx. $15 \mathrm{~cm}^{-1}$ ). For energetically higher transitions, the frequency jump decreases, e.g., the difference between $E_{22}^{S}$ and $E_{11}^{M}$ is approx. $9 \mathrm{~cm}^{-1}$. Although the calculated frequency difference is smaller than experimentally observed, the experimentally observed discontinuity is reproduced in our simulations and is shown to be a conse- quence of the diameter dependence of phonon frequencies in carbon nanotubes.

\section{CONCLUSION}

In summary, we derived a theroretical model to describe the double-resonant scattering process in arbitrary CNTs. We applied this model to describe the diameter dependence of the $D$ mode in carbon nanotubes for the resonant optical transitions. The presented approach is independent of the specific calculational model used for the electronic band structure or phonon dispersion and is therefore universally valid. We showed that, depending on the experimental conditions, in general two different diameter dependences can be observed. Furthermore, we proved that the experimentally observed discontuinity between different transition branches is due to curvature effects that alter the TO phonon dispersion at the $K$ point. Finally, we derived a quantification of the diameter-dependent frequency correction of the $D$ mode in carbon nanotubes with respect to the $D$ mode in graphite. The presented results answer the longstanding question regarding the diameter dependence of the $D$ in carbon nanotubes. Finally, we want to explicitly point out that our approach is equally valid for all other double-resonant Raman modes in CNTs and can be easily expanded to intravalley scattering processes.

\section{ACKNOWLEDGMENTS}

The authors thank I. Milošević (University of Belgrade, Serbia) for useful discussions and kind support with the POLSym code. This work was supported by the European Research Council (ERC) under grant no. 259286 and by the DFG under grant number MA 4079/7-2.
* fhz@physik.tu-berlin.de

1 S. Reich, C. Thomsen, and J. Maultzsch, Carbon Nanotubes: Basic Concepts and Physical Properties (WileyVCH, Weinheim, 2004).

2 A. H. Castro Neto, F. Guinea, N. M. R. Peres, K. S. Novoselov, and A. K. Geim, Rev. Mod. Phys. 81, 109 (2009).

3 R. H. Baughman, A. A. Zakhidov, and W. A. de Heer, Science 297, 787 (2002).

4 S. J. Tans, A. R. M. Verschueren, and C. Dekker, Nature 393, 49 (1998).

5 F. Schedin, A. K. Geim, S. V. Morozov, E. W. Hill, P. Blake, M. I. Katsnelson, and K. S. Novoselov, Nature Materials 6, 652 (2007).

6 A. Hirsch, Angewandte Chemie International Edition 41, 1853 (2002).

7 M. S. Strano, C. A. Dyke, M. L. Usrey, P. W. Barone, M. J. Allen, H. Shan, C. Kittrell, R. H. Hauge, J. M. Tour, and
R. E. Smalley, Science 301, 1519 (2003).

8 J. M. Englert, C. Dotzer, G. Yang, M. Schmid, C. Papp, J. M. Gottfried, H. P. Steinrück, E. Spiecker, F. Hauke, and A. Hirsch, Nature Chemistry 3, 279 (2011).

9 Y. Maeda, K. Saito, N. Akamatsu, Y. Chiba, S. Ohno, Y. Okui, M. Yamada, T. Hasegawa, M. Kako, and T. Akasaka, Journal of the American Chemical Society 134, 18101 (2012).

10 S. Reich and C. Thomsen, Phil. Trans. R. Soc. Lond. A 362, 2271 (2004).

11 F. Tuinstra and J. L. Koenig, J. Chem. Phys. 53, 1126 (1970).

12 C. Thomsen and S. Reich, Phys. Rev. Lett. 85, 5214 (2000).

13 J. Maultzsch, S. Reich, and C. Thomsen, Phys. Rev. B 70, 155403 (2004).

14 J. Maultzsch, S. Reich, and C. Thomsen, Phys. Rev. B 64, 121407 (2001). 
15 M. Damnjanović, I. Milošević, T. Vuković, and R. Sredanović, Phys. Rev. B 60, 2728 (1999).

16 J. Laudenbach, F. Hennrich, H. Telg, M. Kappes, and J. Maultzsch, Phys. Rev. B 87, 165423 (2013).

17 A. G. Souza Filho, A. Jorio, G. Dresselhaus, M. S. Dresselhaus, R. Saito, A. K. Swan, M. S. Ünlü, B. B. Goldberg, J. H. Hafner, C. M. Lieber, and M. A. Pimenta, Phys. Rev. B 65, 035404 (2001).

18 M. A. Pimenta, A. Jorio, S. D. M. Brown, A. G. Souza Filho, G. Dresselhaus, J. H. Hafner, C. M. Lieber, R. Saito, and M. S. Dresselhaus, Phys. Rev. B 64, 041401 (2001).

19 A. G. Souza Filho, A. Jorio, G. G. Samsonidze, G. Dresselhaus, M. A. Pimenta, M. S. Dresselhaus, A. K. Swan, M. S. Ünlü, B. B. Goldberg, and R. Saito, Phys. Rev. B 67, 035427 (2003).

${ }^{20}$ M. Damnjanović, I. Milošević, E. Dobardžić, T. Vuković, and B. Nikolić, "Applied Physics of Nanotubes; Fundamentals of Theory, Optics and Transport Devices," (Springer, Berlin, 2005) Chap. 2.

21 A. Grüneis, J. Serrano, A. Bosak, M. Lazzeri, S. L. Molodtsov, L. Wirtz, C. Attaccalite, M. Krisch, A. Rubio, F. Mauri, and T. Pichler, Phys. Rev. B 80, 085423 (2009).

22 J. Kürti, V. Zólyomi, A. Grüneis, and H. Kuzmany, Phys. Rev. B 65, 165433 (2002).

23 J. Laudenbach, F. Hennrich, M. Kappes, and J. Maultzsch, physica status solidi (b) 249, 2460 (2012).

24 P. Venezuela, M. Lazzeri, and F. Mauri, Phys. Rev. B 84, 035433 (2011).

25 M. Damnjanović, T. Vuković, and I. Milošević, J. Phys. A: Math. Gen. 33, 6561 (2000).

${ }^{26}$ J. Maultzsch, S. Reich, C. Thomsen, E. Dobardžić, I. Milošević, and M. Damnjanović, Solid State Communications 121, 471 (2002).

27 See the Supplemental Material at http: which includes Refs. 1, 15, 25, 26, 32, 34, 37, 39-45.

28 V. N. Popov and L. Henrard, Phys. Rev. B 70, 115407 (2004).

29 O. Gülseren, T. Yildirim, and S. Ciraci, Phys. Rev. B 65, 153405 (2002).

30 V. N. Popov, New Journal of Physics 6, 17 (2004).

31 H. Kataura, Y. Kumazawa, Y. Maniwa, I. Umezu, S. Suzuki, Y. Ohtsuka, and Y. Achiba, Synthetic Metals 103, 2555 (1999).

32 J. Laudenbach, Ph.D. thesis, TU Berlin (2014), urn:nbn:de:kobv:83-opus4-46019.

33 J. Laudenbach, D. Schmid, F. Herziger, F. Hennrich, M. Kappes, M. Muoth, M. Haluska, F. Hof, C. Backes, F. Hauke, A. Hirsch, and J. Maultzsch, submitted (2015).

${ }^{34}$ H. Telg, J. G. Duque, M. Staiger, X. Tu, F. Hennrich, M. M. Kappes, M. Zheng, J. Maultzsch, C. Thomsen, and S. K. Doorn, ACS Nano 6, 904 (2012).

35 J. Kürti, G. Kresse, and H. Kuzmany, Phys. Rev. B 58, R8869 (1998).

${ }^{36}$ V. N. Popov and P. Lambin, Phys. Rev. B 73, 085407 (2006).

37 H. Telg, J. Maultzsch, S. Reich, F. Hennrich, and C. Thomsen, Phys. Rev. Lett. 93, 177401 (2004).

38 A. C. Ferrari and D. M. Basko, Nature Nanotech. 8, 235246 (2013).

39 R. Jishi, L. Venkataraman, M. Dresselhaus, and G. Dresselhaus, Chem. Phys. Lett. 209, 77 (1993).
40 J. Maultzsch, H. Telg, S. Reich, and C. Thomsen, Phys. Rev. B 72, 205438 (2005).

${ }^{41}$ H. Telg, J. Maultzsch, S. Reich, and C. Thomsen, Phys. Rev. B 74, 115415 (2006).

42 M. Machón, S. Reich, H. Telg, J. Maultzsch, P. Ordejón, and C. Thomsen, Phys. Rev. B 71, 035416 (2005).

43 S. D. Costa, C. Fantini, A. Righi, A. Bachmatiuk, M. H. Rmmeli, R. Saito, and M. A. Pimenta, Carbon 49, 4719 (2011).

44 E. Di Donato, M. Tommasini, C. Castiglioni, and G. Zerbi, Phys. Rev. B 74, 184306 (2006).

45 V. N. Popov, V. E. Van Doren, and M. Balkanski, Phys. Rev. B 59, 8355 (1999). 


\title{
Supplementary material for the paper:
}

\section{Understanding double-resonant Raman scattering in chiral carbon nanotubes: Diameter and energy dependence of the $D$ mode}

\author{
Felix Herziger, ${ }^{1, *}$ Asmus Vierck, ${ }^{1}$ Jan Laudenbach, ${ }^{1}$ and Janina Maultzsch ${ }^{1}$ \\ ${ }^{1}$ Institut für Festkörperphysik, Technische Universität Berlin, Hardenbergstrasse 36, 10623 Berlin, Germany
}

\section{Helical wave-vector backfolding}

Since this paper mainly considers chiral carbon nanotubes, it is more convenient to use the helical representation of the wavevector and electronic bands instead of the conventional linear representation ${ }^{1,2}$. Therefore, we first give a short introduction and motivation to the helical quantum numbers. We will show that the electronic band structure in helical space is given by the cut of the helical wavevector with the electronic $\pi$ and $\pi^{*}$ bands along its path in reciprocal space.

In linear quantum numbers, the electronic bands are given along the quasi-continuous reciprocal vector $\mathbf{k}_{z}$, which has the form

$$
\mathbf{k}_{z}=-\frac{n_{2}}{q} \mathbf{k}_{1}+\frac{n_{1}}{q} \mathbf{k}_{2}
$$

Here, $\mathbf{k}_{1}$ and $\mathbf{k}_{2}$ are the unit vectors in reciprocal space [compare Fig. 1 (a)] and $n_{1}$ and $n_{2}$ are the chiral indices of the tube. The number of graphene hexagons per unit cell is $q$, which also gives the number of electronic subbands ${ }^{3}$. In linear space the vector $\mathbf{k}_{z}$ is restricted to the interval $(-\pi / a, \pi / a]$, where the brackets ( and ] indicate open and closed interval limits, respectively. Here, $a$ is the translational period along the tube axis. The electronic dispersion of the $q$ subbands is then given by $q$ cuts of $\mathbf{k}_{z}$ with the $\pi / \pi^{*}$ bands of graphene. However, $q$ increases rapidly even for non-high-index tubes, e.g., $q=386$ for a $(9,7)$ tube. Therefore, the linear representation becomes complicated very quickly. The transformation from the linear to the helical representation resolves this problem and is therefore much more convenient for displaying band structures or phonon dispersion relations ${ }^{2}$.

If $\mathbf{k}_{z}$ is transformed into its helical representation $\widetilde{\mathbf{k}}_{z}$, the interval is extended by the factor $q / n$, where $n$ is greatest common divisor of $\left(n_{1}, n_{2}\right) .{ }^{4}$ This means that the $q$ subbands in linear space are "rearranged" to form $n$ subbands in helical space. Thus, the helical vector is defined in the interval $(-q \pi / n a, q \pi / n a]$ and has the form ${ }^{1}$

$$
\widetilde{\mathbf{k}}_{z}=\frac{1}{n}\left(-n_{2} \mathbf{k}_{1}+n_{1} \mathbf{k}_{2}\right)
$$

As before, the electronic band structure of CNTs in the helical representation is given by the $n$ cuts of the helical vector with the $\pi / \pi^{*}$ bands. The different subbands are labelled with the helical quantum number $\widetilde{m}$. In contrast to the linear represenation, the prefactors of $\mathbf{k}_{1}$ and $\mathbf{k}_{2}$ in the helical image are by defintion always integers, simply $n_{1}$ and $n_{2}$. This means that the $\widetilde{m}=0$ subband always connects two $\Gamma$ points on the reciprocal hexagonal grid, which are given by $\Gamma=(0,0)$ and $\Gamma_{1}=\left(-n_{2} / n, n_{1} / n\right)$ in units of the reciprocal lattice vectors. Hence, the length of the helical vector depends solely on $\left(n_{1}, n_{2}\right)$ and is therefore directly proportional to the diameter of a carbon nanotube. This result is depicted in Fig. 1 (a) for two different chiral tubes. It is more convenient to use the helical representation of the electronic bands, especially for coprime tubes $(n=1)$, since all information about the electronic dispersion is given in just one subband. For tubes with more than one electronic subband $(n>1)$, the other subbands can be found by performing a parallel translation of the helical vector. A detailed analysis how of to obtain the shifted $\widetilde{m} \neq 0$ subbands can be found in Ref. 1 .

Next, we want to introduce the concept of backfolding the helical band structure of carbon nanotubes. This approach is closely related to the usual sampliing og the Brillouin zone that is already known for linear bands in CNTs ${ }^{4,5}$, but was never applied to their helical bands. In Fig. 1(a) we illustrate the folding of helical bands for two CNTs: for a $(5,3)$-tube, where $\widetilde{\mathbf{k}}_{z}$ runs close to the armchair direction - thus introduced here as an "armchair-like" tube (ACL) and for a $(4,1)$-tube, named "zig-zag-like" (ZZL) accordingly. The helical band of an ACL tube can now be folded back along the dashed lines running through $\Gamma$ points in zig-zag direction, and vice versa for ZZL tubes along those in armchair direction. Using this approach, the helical vector along $\Gamma-\Gamma_{1}$ (black line inside the blue shaded rectangle) can be reduced to the sawtooth-like path along $\Gamma-\Gamma_{2}$ (red line inside the red shaded rectangle). While both paths are of course equivalent, it becomes apparent that the amount of hexagons covered by the helical vector reduces significantly and the location of minima becomes even more obvious. 

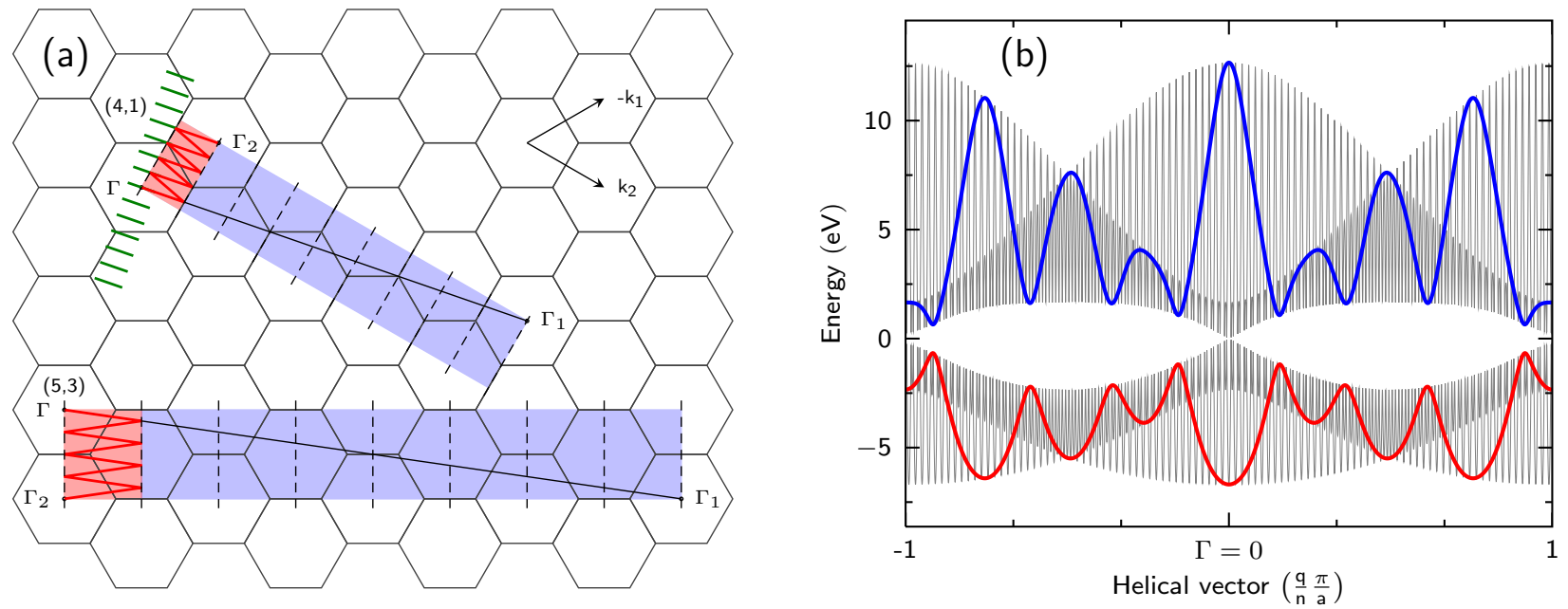

Figure 1. (a) Backfolding of the helical band structure of single-walled carbon nanotubes. The band structure of the "armchairlike" $(5,3)$-tube is projected onto the zig-zag direction, whereas the "zig-zag-like" (4,1)-tube is projected onto the armchair direction. The band structures along the $\Gamma$ - $\Gamma_{1}$-line can be represented by the folded path along $\Gamma-\Gamma_{2}$. For the $(4,1)$ tube, we also show the linear bands (green). (b) Helical band structure of $(99,97)$-tube (gray, background) and a (5,3)-tube (thick lines, foreground). $q$ is the number of hexagons per unit cell, $n$ is the greatest common divisor of $\left(n_{1}, n_{2}\right)$, and $a$ is the translational period of the tube along the axis.

The consequences of the backfolding become more clear, when we consider the limit of large-diameter tubes, e.g., a $(99,97)$-tube. As shown before, the length of the helical vector is directly proportional to the tube diameter. Consequently, the helical vector gets folded at an increased number of positions for large tubes. In the following, we want to refer to this folding as the sampling of the Brillouin zone. Since for the ACL tubes $(5,3)$ and $(99,97) n_{2}$ equals $n_{1}-2$, in both cases the zig-zag component of their helical vector will span cross two "rows" of hexagons and thus the projection area for both tubes is the same (see Fig. 1). Hence, the folded path in the red-shaded rectangle must cover the same three underlying hexagons more densely for the $(99,97)$-tube compared to the $(5,3)$-tube. Naturally, this means that the electronic $\pi$ bands of the graphene analogon are simply sampled more closely. This effect can be nicely seen in Fig. 1 (b), which shows the helical band structure of both tubes along their normalized helical vector. Here it becomes obvious that the band structure of the (99,97)-tube simply corresponds to a denser sampling of the same area as in case of the (5,3)-tube. Since both projection areas are equivalent, both band structures could also be imagined encased in an evenlope function representing the band structure of the three graphene hexagons in their projection area. In our example, there are three $\Gamma, M$ and $K$ points in the projection area [compare Fig. 1 (a)], which can be seen in the band structure of the $(99,97)$-tube very nicely. With increasing sampling, the folded helical vector cuts the Brillouin zone more often and hence also closer to the $K$ points. Since the energetically lowest transitions are those close to the $K$-Point, it is clear that the transition energy becomes smaller for larger tubes. This is a well-known result $^{4}$ and can be seen in our model very easily.

\section{Systematics of the $D$-mode frequencies for non-coprime CNTs}

We now want to extend the geometrical model presented in the main manuscript to non-coprime tubes, which have more than one subband. The situation is shown in Fig. 2 for a $(15,3)$-tube, which has three subbands $(\widetilde{m}=-1,0,+1)$. Due to the scattering between equivalent minima and the point symmetry at the $\Gamma$ point, the scattering process can always be represented by a scattering across the $\Gamma$ point at $(0,0)$. Let us now assume that an electron is scattered between a minimum on the $\widetilde{m}=+1$ band to a minimum on the $\widetilde{m}=-1$ band, i.e., the electron is scattered between $-k_{\min }$ and $k_{\min }$. The resonant phonon wave vector is given by $q_{\mathrm{res}}=2 k_{\min }$. We can now draw the already introduced triangles with the same edge points as in the case for coprime tubes. Hence, excactly the same systematics as before apply. Furthermore, our geometrical approach instrincally assigns the phonons from the correct band, i.e., with the correct quantum number. In our example the electrons gets scattered between the $\widetilde{m}=+1$ and $\widetilde{m}=-1$ band, which results in a change of the helical quantum number by $\Delta \widetilde{m}=-2$. Therefore, the phonons must stem from the $\widetilde{m}=-2$ band, which is equivalent to the $\widetilde{m}=+1$ band. Since the $\widetilde{m}$ bands are equidistantly spaced in the reciprocal lattice, this result is naturally produced in our model and can be seen very nicely. 


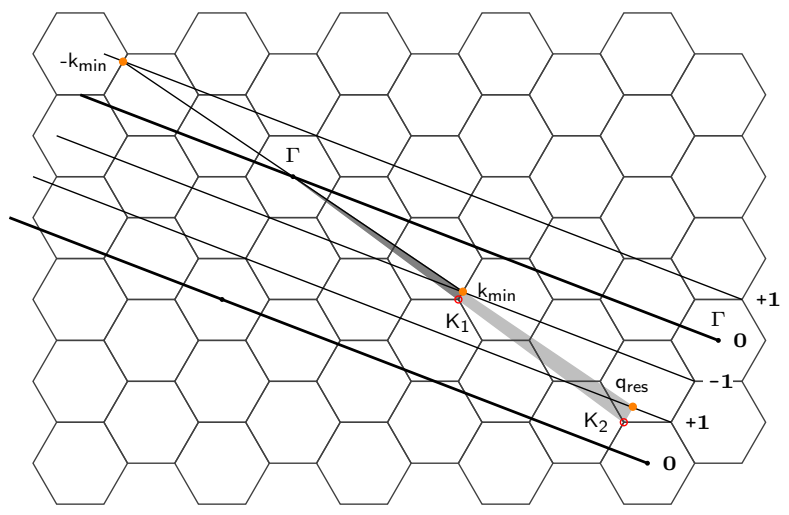

Figure 2. Illustration of the geometrical model for non-coprime tubes. In this example, we used the helical bands of a (15,3)tube. The numbers $-1,0,+1$ represent the helical quantum number $\widetilde{m}$ of each subband. Due to the scattering between two equivalent minima and the point symmetry at the $\Gamma$ point, the resonant phonon wave-vector is always twice the distance $\Gamma-k_{\min }$. Hence, the same systematics as before apply.
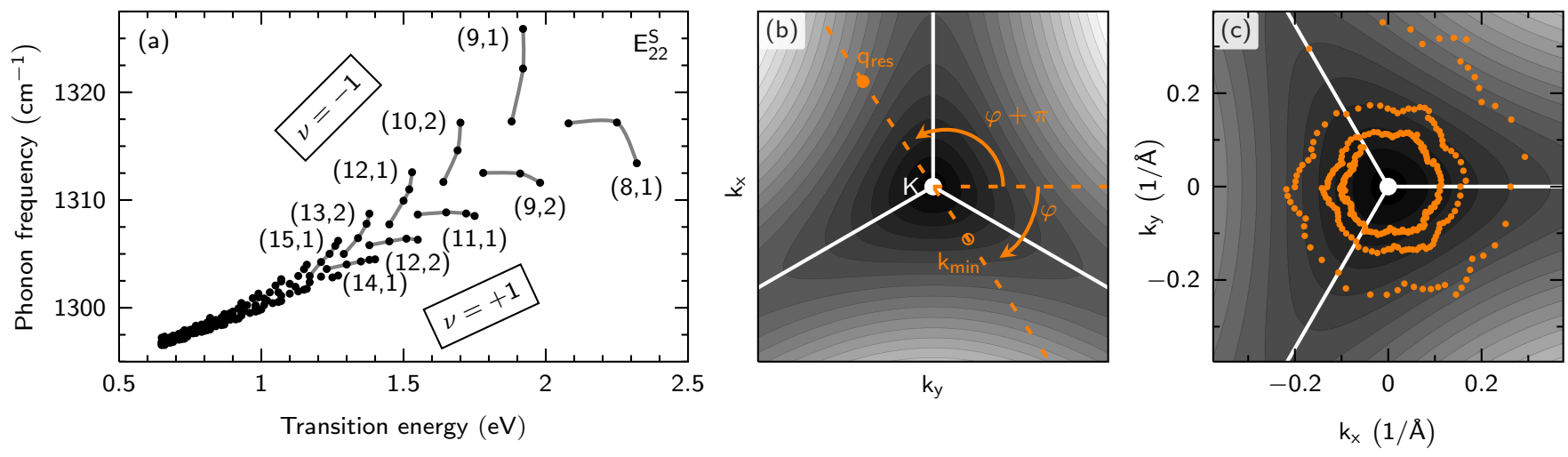

Figure 3. (a) Family-behavior of the $D$-mode frequencies for $\mathrm{E}_{22}^{S}$ transition. The labels refer to the chiral indices of the outmost tube of each branch. (b) Reduced scheme of the geometric model at a single $K$ point. $k_{\text {min }}$ and $q_{\text {res }}$ label the positions at which the electronic transition occurs and the resonant phonons stem from, respectively. The solid white lines denote the $K-M$ high-symmetry directions. (c) Calculated resonant phonon wave-vectors for the $\mathrm{E}_{22}^{S}$ transition projected on a single $K$ point at $(0,0)$. The positions of the resonant phonon wave vectors evolve along a spiral into the $K$ point for increasing tube diameter.

\section{A. $\quad D$-mode families}

The family-behavior for Raman modes in carbon nanotubes is already known for the radial-breathing modes ${ }^{6,7}$, where each branch is given by the relation

$$
\nu=\bmod \left(n_{1}-n_{2}\right) 3= \pm 1 \text { and } \mu=2 n_{1}+n_{2}=\text { const. }
$$

Here, we will show that the $D$ mode follows a similar behavior and that the $D$-mode frequencies arrange in a Katauraplot-like pattern. Figure 3 (a) shows the calculated $D$-mode frequencies for the $E_{22}$ transition of semiconducting tubes (taken from Fig. 3 of the main manuscript). As can be easily seen, the frequencies are arranged in branches, which are given by above mentioned relation. The labels in Fig. 3 (a) refer to the chiral indices of the outmost tube of each branch. Compared to the RBM-families, the positions of the $D$-mode branches are flipped, i.e., the $\nu=-1$ families are on the upper side of the $E_{22}^{S}$ branch and the $\nu=+1$ families on the lower side. This behavior can be understood if we reduce our geometrical model to just a single $K$ point.

If the electronic transition occurs at a distance $h$ and an angle $\varphi$ near a $K$ point, the resonantly enhanced phonons stem from the distance $2 h$ and an angle $\varphi$ from a $K^{\prime}$ point (see Fig. 2). Since $K$ and $K^{\prime}$ are equivalent but rotated by $180^{\circ}$ compared to each other, a phonon with distance $2 h$ and angle $\varphi$ at $K^{\prime}$ can be represented by a phonon with the same distance but angle $\varphi+\pi$ at $K$. Thus, we can reduce the electronic transition and the corresponding resonant phonons to a single $K$ point, where the electronic transition and the resonantly enhanced phonon stem from oppsite sides. Figure $3(\mathrm{~b})$ shows the schematics of the reduced model. From the Kataura plot, we know that the electronic 


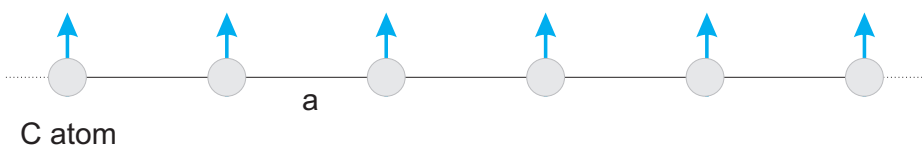

(a)
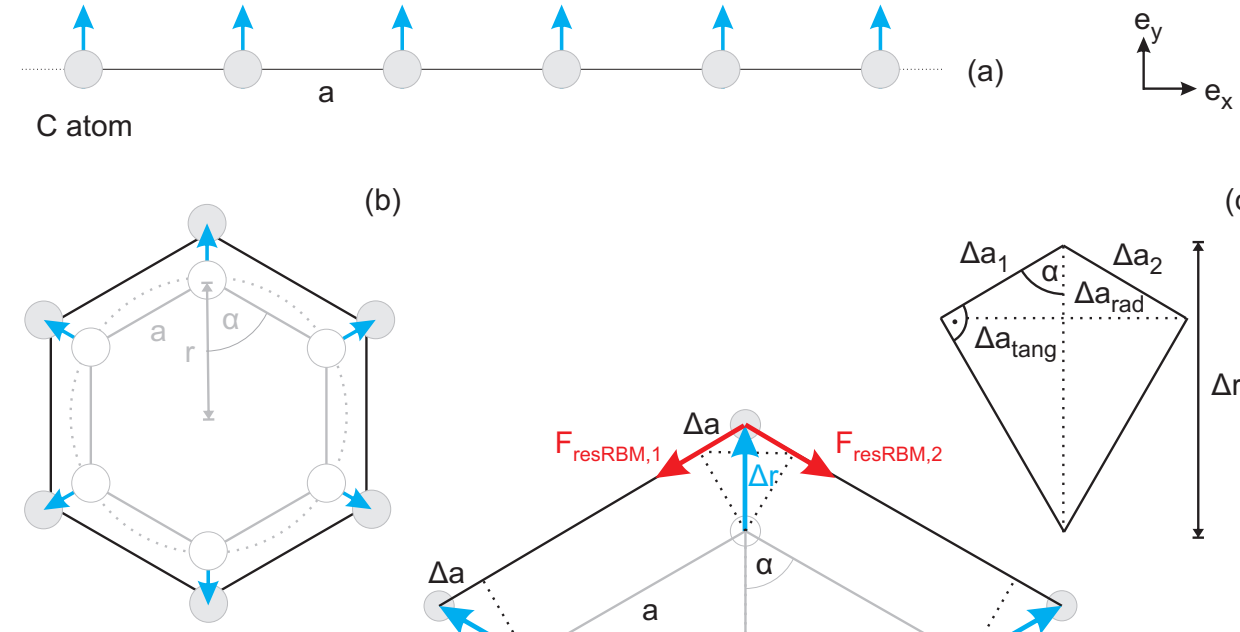

(b)

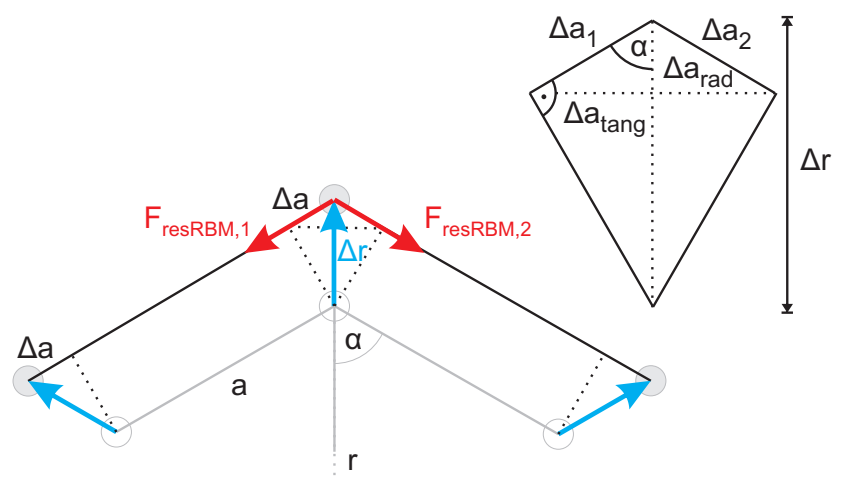

Figure 4. Sketch of the restoring forces induced by curvature for the RBM. (a) Model of a nanotube with infinite diameter $d$. All carbon atoms move perpendicular to the sigma bonds $a$. (b) Model of a nanotube with radius $r=d / 2$ in the position of equilibrium (open circles) and after displacement (gray circles). All atoms move in radial direction. (c) Zoom to an atom with its closest neighbors. The restoring forces are drawn in red. Figure taken from Ref. 13.

transitions split up into branches with $\nu= \pm 1$ families. Thus, also the resonant phonons for the $D$-mode scattering process split up accordingly. This can be also seen in Fig. 3 (c), where we plot the resonantly enhanced phonons for the $\mathrm{E}_{22}^{S}$ transition. As can be seen, the phonons are arranged in branches forming a spiral that runs into the $K$ point with increasing tube diameter. Thus, tubes with small diameters can be found in the outer region, meaning a higher $D$-mode frequency. If we now want to understand the family behavior of the $D$ mode, we have to keep in mind that we plot the resonantly enhanced phonons, which are on the $K$-point side opposite to the electronic transition. Thus, the phonon frequencies behave contrary to the families of the electronic transitions or the RBMs. Since the TO phonon branch exhibits a trigonal warping at $K$ that is opposite to the electronic trigonal warping, the splitting is not as pronounced as for the RBM families. Moreover, the splitting is barely visible for the $E_{11}^{S}$ transition since the trigonal warping vanishes in vicinity of the $K$ point.

For the RBM families, it is well-known that the $\nu=-1$ family of the $E_{22}^{S}$ transition gives a much larger Raman signal compared to the $\nu=+1$ family $^{8}$. This behaviour is due to a higher oscillator strength of the optical transition and a higher electron-phonon coupling for these tubes ${ }^{9}$. Although the electron-phonon coupling might be different for the TO phonon, the oscillator strength should be the same for the RBM and the $D$-mode scattering process. Thus, a similar behaviour regarding the intensities of the $D$-mode families can be expected. This would mean that the upper family of the $D$-mode $E_{22}^{S}$ branch is dominant in an experiment and the lower family gives a much weaker in signal.

\section{Curvature effect on phonon frequencies in single-walled carbon nanotubes}

In order to understand the dependence of Raman-mode frequencies on the CNT diameter, we introduce a simple spring model of a nanotube. The use of a spring model is justified by the results from experiments with ${ }^{13} \mathrm{C}$ isotopes ${ }^{10}$ and calculations using force constant models $2,5,11,12$. The structure of a nanotube is simply approximated by a closed ring of carbon atoms. This simple model will be sufficient to simulate the inversely-proportional relation between diameter and RBM frequency and the proportional relation between diameter and $G^{-}$-mode frequency (more precisely, the $G^{-}$mode related to the TO phonon branch for semiconducting nanotubes). Furthermore, this model will give the right magnitude of the frequency shift due to curvature. This leads to a general understanding of the dependence of frequency on diameter for in-plane and out-of-plane vibrations.

A nanotube with an infinite diameter can be approximated by a chain of carbon atoms with a constant atomic distance $a$. The introduction of different $a$ 's would lead to a more precise model, but cannot be handled with this approach. For this nanotube with infinite diameter, the RBM is an acoustic out-of-plane vibration and therefore represents a translation with zero frequency [Fig. 4 (a)]. 
A nanotube with a given diameter is represented by a closed ring of atoms. Figure 4 (b) shows this for a nanotube with radius $r$ and angle $\alpha$ between radius $r$ and atomic bond $a$. The former, strict vibration orthogonal to the sigma bonds $a$ [Fig. 4 (a)] now becomes the breathing vibration of the nanotube, with a decreased angle $\alpha$ compared to a tube with infinite diameter. Figure 4(c) shows the zoom to an atom with its closest neighbors in the position of equilibrium (open circles) and after displacement (gray circles). In next neighbor approximation, two effective restoring forces $\left(\vec{F}_{\text {resRBM }}\right)$ can be assumed for each atom, marked with red arrows for the upper atom.

In general, the restoring force $\vec{F}$ is proportional to the displacement $\Delta \vec{a}$ of a spring with $\vec{F}=-f \Delta \vec{a}, f$ is the spring constant. For this model, $\Delta \vec{a}$ can be decomposed into a radial part $\Delta \vec{a}_{\text {rad }}$ and tangential part $\Delta \vec{a}_{\text {tang. This leads to }}$ the effective restoring force

$$
\begin{aligned}
\vec{F}_{\mathrm{resRBM}} & =\vec{F}_{\mathrm{resRBM}, 1}+\vec{F}_{\mathrm{resRBM}, 2}=-f\left(2 \Delta \vec{a}_{1}+2 \Delta \vec{a}_{2}\right) \\
& =-2 f\left(\Delta \vec{a}_{\mathrm{rad}, 1}+\Delta \vec{a}_{\mathrm{tang}, 1}+\Delta \vec{a}_{\mathrm{rad}, 2}+\Delta \vec{a}_{\mathrm{tang}, 2}\right) \\
& =2 f\left[2 \Delta a_{\mathrm{rad}}\left(-\vec{e}_{y}\right)\right]=f \Delta a \cdot 4 \cos (\alpha) \cdot\left(-\vec{e}_{y}\right) .
\end{aligned}
$$

The effective force constant $f^{*}$ can be found by dividing the effective restoring force $\vec{F}_{\text {resRBM }}$ by the displacement of the atom $\Delta r$ in radial direction. With $\Delta r \cos (\alpha)=\Delta a$ it follows

$$
f_{\mathrm{RBM}}^{*}(\alpha)=\frac{\left|\vec{F}_{\text {resRBM }}\right|}{|\Delta r|}=4 f \cos (\alpha)^{2} .
$$

The effective force constant $f^{*}$ changes with $\alpha$, which is related to a change in diameter. The relation between $\alpha$ and diameter $d$ can be found with the cosine formula

$$
\alpha(d)=\arccos \left(\frac{a}{2 r}\right)=\arccos \left(\frac{a}{d}\right) .
$$

Insertion of Eq. (7) in Eq. (6) gives the dependence of the RBM frequency on diameter:

$$
\omega_{\mathrm{RBM}}(d)=\sqrt{\frac{f_{\mathrm{RBM}}^{*}}{m}}=2 \sqrt{\frac{f}{m}} \cdot \frac{a}{d} .
$$

This equation reflects the well known $1 / d$ behavior for the RBM frequency.

Based on the same considerations and thoughts for Fig. 5 the effective restoring force of the $G^{-}$mode (TO phonon mode) can be determined to

$$
\vec{F}_{\mathrm{res} G^{-}}=f \Delta a \cdot 4 \sin (\alpha) \cdot\left(\vec{e}_{x}\right) .
$$

With $\Delta t \sin (\alpha)=\Delta a$ the effective spring constant $f^{*}$ can be calculated to:

$$
f_{G^{-}}^{*}(\alpha)=\frac{\left|\vec{F}_{\mathrm{res} G^{-}}\right|}{|\Delta t|}=4 f \sin (\alpha)^{2} .
$$

Insertion of Eq. (7) in Eq. (10) and using the relation $\sin [\arccos (x)]=\sqrt{1-x^{2}}$ the dependence of the $G^{-}$-mode frequency on diameter can be found:

$$
\omega_{G^{-}}(d)=\sqrt{\frac{f_{G^{-}}^{*}}{m}}=2 \sqrt{\frac{f}{m}} \cdot \sqrt{1-\left(\frac{a}{d}\right)^{2}} .
$$

Without curvature, the frequency of the $G^{-}$mode must be equal to the frequency of the $G^{+}$mode (LO phonon mode), so $2 \sqrt{f / m}$ can be assigned to $1591 \mathrm{~cm}^{-1}$. The distance to closest neighbors is set to $a=0.213 \mathrm{~nm}$, which is the mean value for the largest and lowest possible distance of carbon atoms in a graphene hexagon.

Figure 6 shows the calculated diameter dependence (red) for the RBM and Fig. 7 for the $G^{-}$mode, together with experimental data (blue). The decrease of the RBM frequency for a diameter range between $0.6-1.2 \mathrm{~nm}$ is $49 \%$ for the calculations and $47 \%$ for the experiment. For the $G^{-}$mode, the increase of frequency for a diameter range between $0.6-1.2 \mathrm{~nm}$ is $5 \%$ for the calculations and $4 \%$ for the experiment. The large difference in absolute frequencies for the RBM between calculation and experiment can be explained by an overestimation of $2 \sqrt{f / m}=1591 \mathrm{~cm}^{-1}$. It is known that the force constant in graphene (no curvature) is weaker for out-of-plane vibrations than for in-plane vibrations ${ }^{5}$.

Overall, a very good agreement between experiment and theory is obtained. For this model, the main effect of curvature on frequency is a change in the angle $\alpha$, related to a cosine function for the RBM (out-of-plane vibration) 


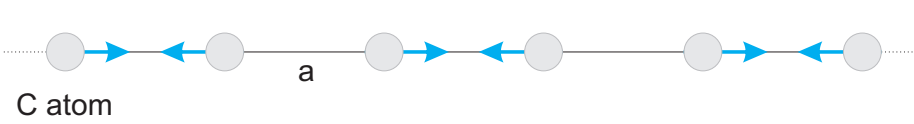

(b)
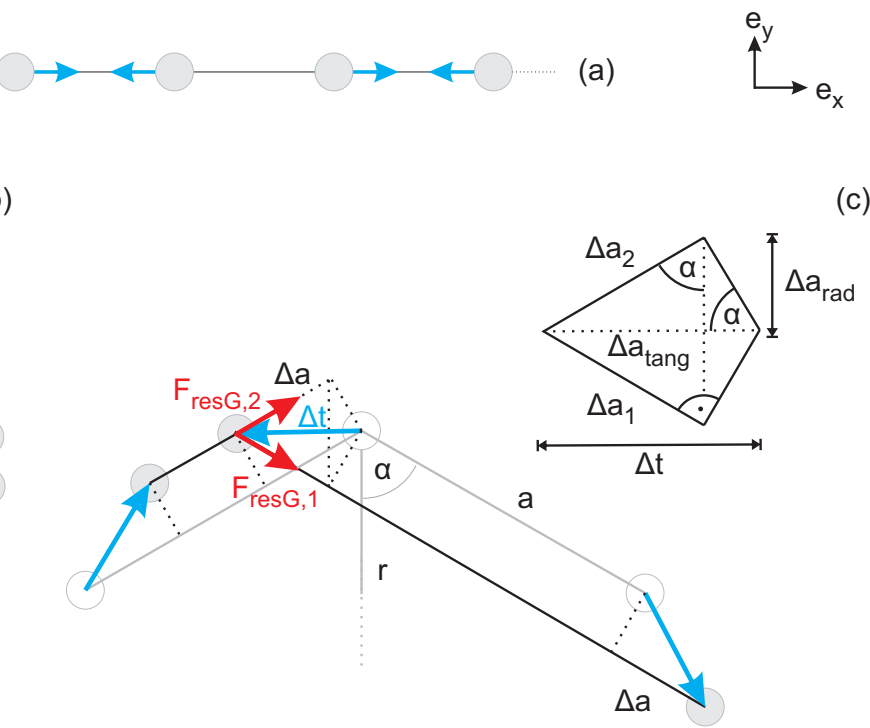

Figure 5. Sketch of the restoring forces induced by curvature for the $G^{-}$mode. (a) Model of a nanotube with infinite diameter $d$. All carbon atoms move parallel to the sigma bonds $a$. (b) Model of a nanotube with radius $r=d / 2$ in the position of equilibrium (open circles) and after displacement (gray circles). All atoms move tangential to the tubes wall. (c) Zoom to an atom with its closest neighbors. The restoring forces are drawn in red. Figure taken from Ref. 13.

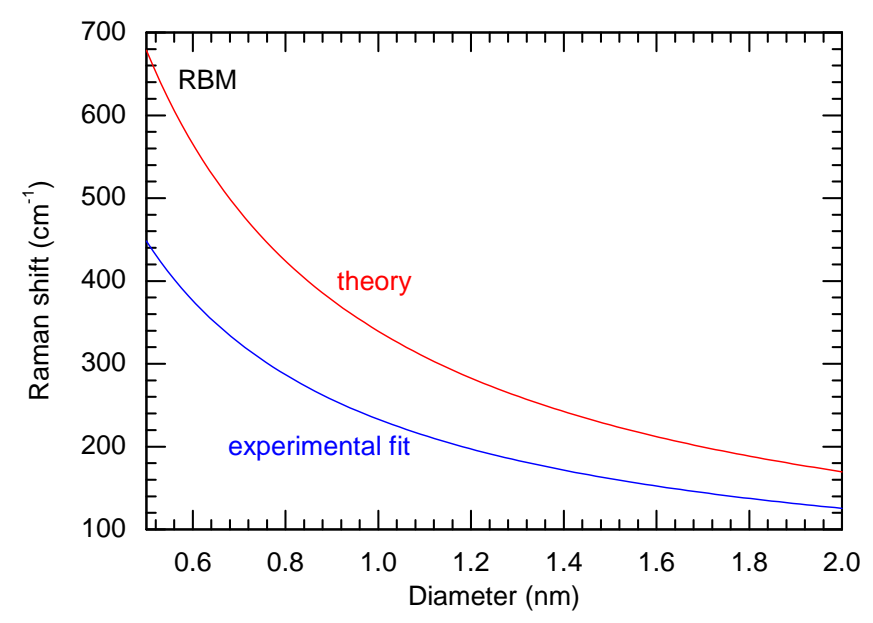

Figure 6. Calculated dependence of RBM-frequency on diameter (red) from Eq. (8) with $a=0.213 \mathrm{~nm}$ and $\sqrt{f / m}=795.5 \mathrm{~cm}{ }^{-1}$. For comparison, fit of experimental data (blue) from ${ }^{7}$ is shown. Figure taken from Ref. 13.

and a sine function for the $G^{-}$mode (in-plane vibration). This leads to a specific change of the effective spring constant and explains the opposite relation between diameter and frequency for RBM and $G^{-}$mode and the weaker influence of the curvature on frequency shift for the $G^{-}$mode in comparison to the RBM. Since for the $D$ mode the atoms move in-plane direction (breathing-like vibration of the carbon hexagons), a similar dependence of the $D$-mode frequency on diameter can be assumed as for the $G^{-}$mode. Of course, curvature-induced strain, changes in the atomic bond lengths and the rehybridization of $s p^{2}$ to $s p^{3}$ orbitals ${ }^{4}$ might affect the frequency as well, but seem not to be the main influence for nanotubes bigger than $0.6 \mathrm{~nm}$.

* fhz@physik.tu-berlin.de

1 M. Damnjanović, T. Vuković, and I. Milošević, J. Phys. A: Math. Gen. 33, 6561 (2000). 


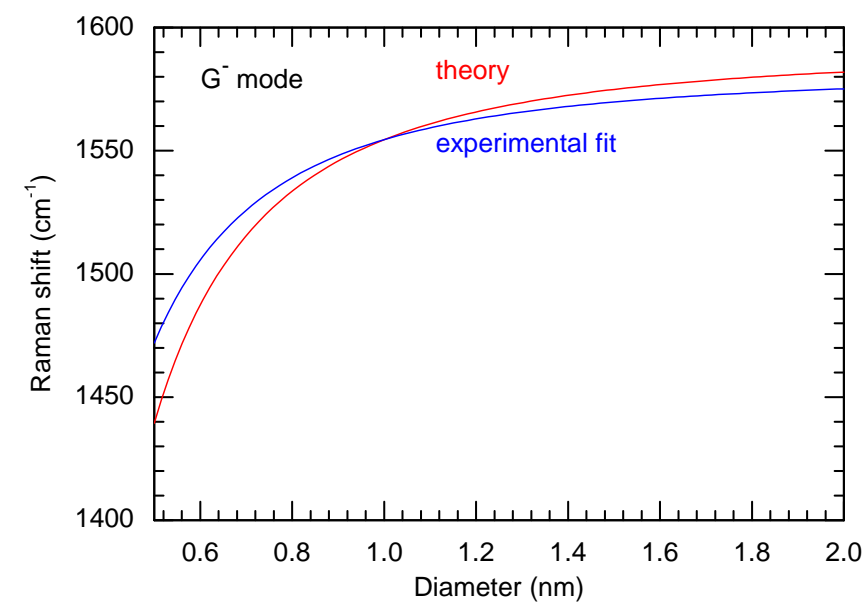

Figure 7. Calculated dependence of $G^{-}$-mode frequency on diameter (red) from Eq. (11) with $a=0.213 \mathrm{~nm}$ and $\sqrt{f / m}=$ $795.5 \mathrm{~cm}^{-1}$. For comparison, fit of experimental data (blue) from ${ }^{14}$ is shown. Figure taken from Ref. 13.

2 J. Maultzsch, S. Reich, C. Thomsen, E. Dobardžić, I. Milošević, and M. Damnjanović, Solid State Communications 121, 471 (2002).

3 M. Damnjanović, I. Milošević, T. Vuković, and R. Sredanović, Phys. Rev. B 60, 2728 (1999).

${ }^{4}$ S. Reich, C. Thomsen, and J. Maultzsch, Carbon Nanotubes: Basic Concepts and Physical Properties (Wiley-VCH, Weinheim, 2004).

${ }^{5}$ R. Jishi, L. Venkataraman, M. Dresselhaus, and G. Dresselhaus, Chem. Phys. Lett. 209, 77 (1993).

${ }^{6}$ H. Telg, J. Maultzsch, S. Reich, F. Hennrich, and C. Thomsen, Phys. Rev. Lett. 93, 177401 (2004).

7 J. Maultzsch, H. Telg, S. Reich, and C. Thomsen, Phys. Rev. B 72, 205438 (2005).

${ }^{8}$ H. Telg, J. Maultzsch, S. Reich, and C. Thomsen, Phys. Rev. B 74, 115415 (2006).

${ }^{9}$ M. Machón, S. Reich, H. Telg, J. Maultzsch, P. Ordejón, and C. Thomsen, Phys. Rev. B 71, 035416 (2005).

10 S. D. Costa, C. Fantini, A. Righi, A. Bachmatiuk, M. H. Rümmeli, R. Saito, and M. A. Pimenta, Carbon 49, 4719 (2011).

11 E. Di Donato, M. Tommasini, C. Castiglioni, and G. Zerbi, Phys. Rev. B 74, 184306 (2006).

12 V. N. Popov, V. E. Van Doren, and M. Balkanski, Phys. Rev. B 59, 8355 (1999).

13 J. Laudenbach, Ph.D. thesis, TU Berlin (2014), URN = urn:nbn:de:kobv:83-opus4-46019.

${ }^{14}$ H. Telg, J. G. Duque, M. Staiger, X. Tu, F. Hennrich, M. M. Kappes, M. Zheng, J. Maultzsch, C. Thomsen, and S. K. Doorn, ACS Nano 6, 904 (2012). 